\title{
Human Dectin-1 Deficiency and Mucocutaneous Fungal Infections
}

\author{
Bart Ferwerda, Ph.D., Gerben Ferwerda, Ph.D., M.D., Theo S. Plantinga, M.S., \\ Janet A. Willment, Ph.D., Annemiek B. van Spriel, Ph.D., Hanka Venselaar, M.S., \\ Clara C. Elbers, Ph.D., Melissa D. Johnson, Ph.D., M.D., \\ Alessandra Cambi, Ph.D., Cristal Huysamen, Ph.D., Liesbeth Jacobs, B.S., \\ Trees Jansen, B.S., Karlijn Verheijen, B.S., Laury Masthoff, M.D., \\ Servaas A. Morré, Ph.D., Gert Vriend, Ph.D., David L. Williams, Ph.D., M.D., \\ John R. Perfect, Ph.D., M.D., Leo A.B. Joosten, Ph.D., Cisca Wijmenga, Ph.D., \\ Jos W.M. van der Meer, Ph.D., M.D., Gosse J. Adema, Ph.D., \\ Bart Jan Kullberg, Ph.D., M.D., Gordon D. Brown, Ph.D., \\ and Mihai G. Netea, Ph.D., M.D.
}

From the Department of Internal Medicine and the Nijmegen Institute for Infection, Inflammation, and Immunity (B.F., G.F., T.S.P., L.J., T.J., K.V., L.M., L.A.B.J., J.W.M.M., B.J.K., M.G.N.); the Department of Tumor Immunology and the Nijmegen Center for Molecular Life Sciences (A.B.S., A.C., G.J.A.); and the Center for Molecular and Biomolecular Informatics, Nijmegen Center for Molecular Life Sciences (H.V., G.V.) - all at Radboud University Nijmegen, Nijmegen; the Complex Genetics Section, Department of Medical Genetics, Division of Biomedical Genetics (C.C.E., C.W.), and the Julius Center for Health Sciences and Primary Care (C.C.E.), University Medical Center Utrecht, Utrecht; the Laboratory of Immunogenetics, Vrije Universiteit, Amsterdam (S.A.M.); and the Department of Genetics, University Medical Center Groningen, University of Groningen, Groningen (C.W.) - all in the Netherlands; the Institute of Infectious Disease and Molecular Medicine, University of Cape Town, Cape Town, South Africa (J.A.W., C.H., G.D.B.); the Division of Infectious Diseases and International Health, Duke University Medical Center, Durham, NC (M.D.J., J.R.P.); and the Department of Surgery, East Tennessee State University, Johnson City (D.L.W.). Address reprint requests to Dr. Netea at the Department of Medicine (463), Radboud University, Nijmegen Medical Center, Geert Grooteplein 8, 6525GA Nijmegen, the Netherlands, or at m.netea@aig.umcn.nl.

Drs. B. Ferwerda and G. Ferwerda contributed equally to this article.

N EnglJ Med 2009;361:1760-7.

Copyright () 2009 Massachusetts Medical Society.

\section{S U M M A RY}

Mucocutaneous fungal infections are typically found in patients who have no known immune defects. We describe a family in which four women who were affected by either recurrent vulvovaginal candidiasis or onychomycosis had the early-stop-codon mutation Tyr238X in the $\beta$-glucan receptor dectin- 1 . The mutated form of dectin- 1 was poorly expressed, did not mediate $\beta$-glucan binding, and led to defective production of cytokines (interleukin-17, tumor necrosis factor, and interleukin-6) after stimulation with $\beta$-glucan or Candida albicans. In contrast, fungal phagocytosis and fungal killing were normal in the patients, explaining why dectin-1 deficiency was not associated with invasive fungal infections and highlighting the specific role of dectin-1 in human mucosal antifungal defense.

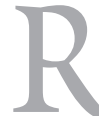

ECURRENT VULVOVAGINAL CANDIDIASIS IS A RELATIVELY COMMON pathological condition, afflicting women of all ages, with more than $90 \%$ of cases caused by C. albicans. ${ }^{1}$ Although the role of diabetes as a predisposing condition has been recognized, most cases occur in healthy women. Recurrent oral, esophageal, or mucocutaneous candidiasis is also diagnosed in some patients without clear predisposing factors. Onychomycosis is a common infection of the nail beds, most often caused by dermatophytes but sometimes caused by C. albicans, that also affects immunocompetent persons. ${ }^{2}$ In contrast, disseminated forms of candida infections are mostly found in patients in whom a defect in neutrophil function is easily recognized or candida species have been introduced in the bloodstream through invasive procedures. ${ }^{3}$ Very little is known about the genetic factors predisposing patients to mucosal or disseminated candida infections.

Recognition of $C$. albicans by the innate host defense system is mediated by pattern-recognition receptors from the toll-like-receptor (TLR) and lectinlike-receptor families. ${ }^{4}$ Mannans from the candida cell wall are recognized by the mannose receptor and TLR4, ${ }^{5}$ and TLR2 recognizes phospholipomannan ${ }^{6}$ and collaborates with the $\beta$-glucan receptor dectin- 1 in the stimulation of cytokine production. ${ }^{7,8}$ 
Recognition of 1,3-linked $\beta$-glucans by dectin- 1 has been shown to be one of the main fungalrecognition pathways, and mice deficient in dectin-1 have an increased susceptibility to C. albicans ${ }^{9}$ and Pneumocystis carinii. ${ }^{10}$ Dectin-1 amplifies TLR2and TLR4-induced cytokine production in both murine and human cells, resulting in the production of cytokines such as tumor necrosis factor, ${ }^{11}$ but also induces signals, independently of the TLRs, for the production of interleukin-17, interleukin-6, and interleukin-10 through a pathway dependent on spleen tyrosine kinase. ${ }^{12}$

\section{METHODS}

\section{INDEX FAMILY AND OTHER SUBJECTS}

To identify functional defects in the immune response to $C$. albicans, we designed a functional screening assay of mononuclear cells isolated from patients with recurrent vulvovaginal candidiasis or oral or esophageal candidiasis. Cells were stimulated with heat-killed C. albicans, and cytokine production after 24 hours was assessed during screening. An immunologic and genetic analysis was performed on mononuclear cells obtained from a patient who had defective cytokine production, on stimulation with C. albicans or $\beta$-glucan, and from four family members. The study protocol was approved by the ethics committee of Nijmegen-Arnhem, the Netherlands. Written informed consent was obtained from all family members studied.

In addition, we studied DNA samples, previously collected to assess the prevalence of the Tyr238X mutations, from several populations in various geographic locations. These samples were obtained from the country of origin, with the exception of the Chinese Han cohort, for which samples were from the Corriell Institute (catalog number, HD1000CHI). For all populations, subjects had provided written informed consent, and study approval was obtained from local ethics committees.

For haplotype analyses, we used genomewide data on single-nucleotide polymorphisms (SNPs) from 1422 whites from the 1958 United Kingdom birth cohort ${ }^{13}$ and from 171 Maasai from Kinyawa, Kenya.${ }^{14}$ Genotype data for the Maasai population were downloaded from the International HapMap Project Web site (www.hapmap.org) and for the white population were obtained with the use of HumanHap300 BeadChips (Illumina). A region of $20 \mathrm{~kb}$ around the SNP rs16910526 was extracted from all samples in both genomewide SNP data sets. We used the Beagle software program to determine the haplotypes from genotypes of all subjects. ${ }^{15}$

\section{GENETIC ANALYSIS}

A detailed description of the methods used to sequence the dectin- 1 gene is presented in the Supplementary Appendix (available with the full text of this article at NEJM.org). Network analysis was performed with the Network program, version 4.5.0.0 (www.fluxus-engineering.com), which uses the median-joining method. ${ }^{16}$ The ancient haplotype was determined with the use of the dbSNP database (www.ncbi.nlm.nih.gov/projects/SNP/). Various analyses were conducted, with weighting applied separately for synonymous changes, nonsynonymous changes, transitions, and transversions.

\section{MODELING OF DECTIN-1 STRUCTURE}

The crystal structure of mouse dectin (Protein Data Bank code 2cl8) was used as a template to build a homology model of the extracellular domain of human dectin-1. Modeling of the extracellular domain was preformed on the "WHAT IF" server (http.//swift.cmbi.ru.nl). Energy minimization and analysis were performed, to obtain the most reliable model of protein folding, with the YASARA program (www.yasara.org/). ${ }^{17}$

\section{FLUORESCENCE-ACTIVATED CELL SORTING}

Human peripheral-blood mononuclear cells (PBMCs) were obtained from the three family members who were homozygous for a dectin-1 mutation, the two family members who were heterozygous for a dectin-1 mutation, and five persons who were homozygous for the wild-type dectin-1 allele. The cells were incubated with murine anti-dectin-1 monoclonal antibody GE2 (5 $\mu \mathrm{g}$ per milliliter $)^{18}$ or isotype-control antibody, followed by allophycocyanin-conjugated goat antimouse antibody (Pharmingen). Dectin-1 expression was determined by means of flow cytometry with the use of a fluorescence-activated cell sorter (FACSCalibur, BD Biosciences). Dectin-1 expression was assessed in cells from a mouse embryonic fibroblast cell line (NIH3T3) after transfection, with the use of an antihemagglutinin antibody (Covance), an IgG1 isotype-control antibody, and an antimouse antibody covalently labeled with R-phycoerythrin (Jackson ImmunoResearch). 


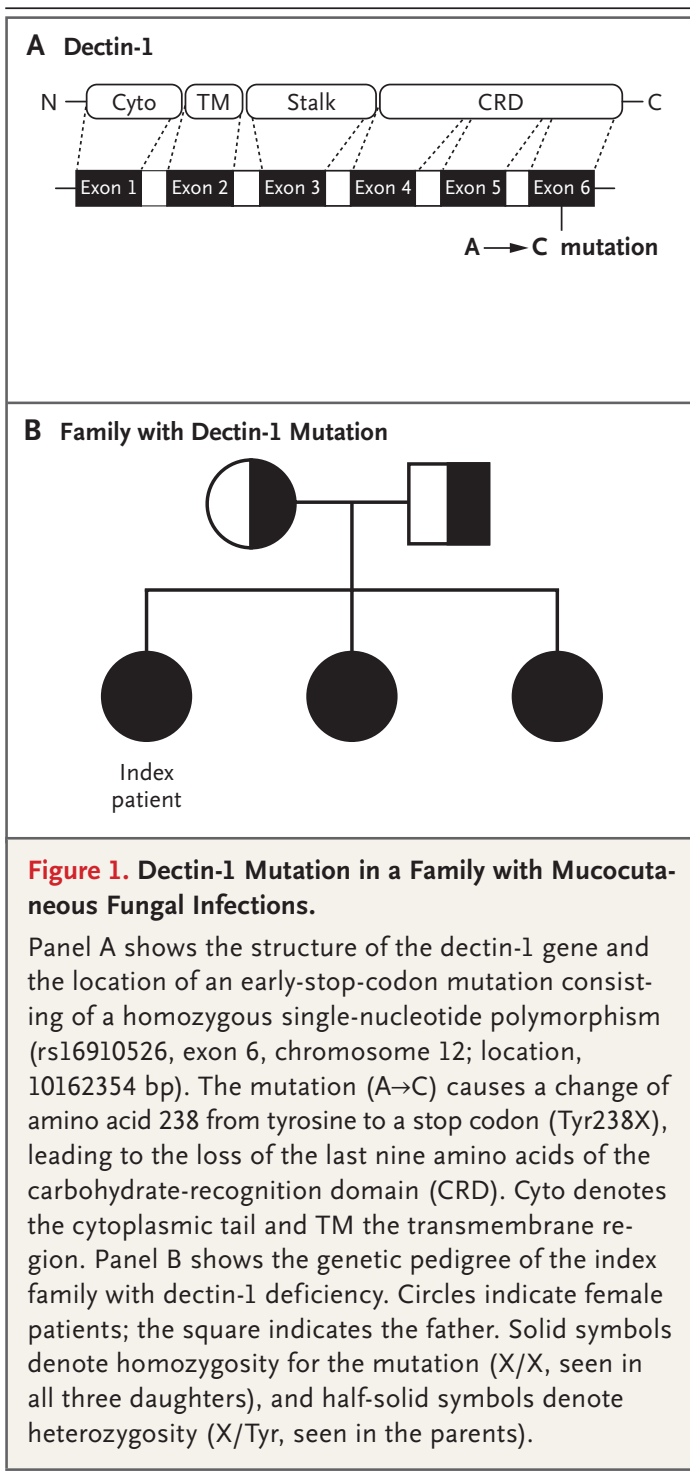

CANDIDA BINDING, PHAGOCYTOSIS, AND FUNGALKILLING ASSAYS

Binding of PBMCs to C. albicans yeast cells was measured by means of flow cytometry. ${ }^{19}$ The phagocytosis and fungal-killing assays for C. albicans were performed as previously described. ${ }^{20}$

\section{CYTOKINE MEASUREMENTS}

$\operatorname{PBMCs}^{12}\left(5 \times 10^{5}\right)$ were incubated with $100 \mu \mathrm{l}$ of $\beta$-glucan (10 $\mu$ g per milliliter) and C. albicans that was live or had been heat-killed (through heating for 30 minutes at $56^{\circ} \mathrm{C}$ ) at a concentration of $1 \times 10^{6}$ yeast cells per milliliter. For interleukin- 17 stimulation assays, PBMCs were stimulated for 5 days in RPMI medium supplemented with $10 \%$ pooled human serum. After 4 hours, 24 hours, or 5 days of incubation at $37^{\circ} \mathrm{C}$, supernatants were collected and stored at $-70^{\circ} \mathrm{C}$ until enzyme-linked immunosorbent assay with the PeliKine-Tool set of reagents (Sanquin).

\section{CLONING AND TRANSFECTION STUDIES}

The human dectin- 1 isoforms were amplified, by means of a polymerase-chain-reaction (PCR) assay, from complementary DNA isolated from samples of peripheral-blood leukocytes obtained from the patients. The two primers used were 5'AAAGGATCCAGGGGCTCTCAAGAACAATG- $3^{\prime}$ and 5'-AAACTCGAGTCTTCCACCCTTCCCCTTAC-3'. The PCR products were purified with the use of the QIAquick PCR purification kit (Qiagen), cloned into the pCR4-TOPO3.1 vector (Invitrogen), and sequenced. The wild-type and mutant dectin-1 were cloned with the use of reverse primers 5'-CCCTTCCTCGAGCATTGAAAACTTC-3' and 5'-AAATCTCGAGTGAGGGCAGACTAC-3', respectively - allowing for the in-frame cloning of a hemagglutinin tag, which does not affect $\beta$-glucan binding. ${ }^{7}$ The products were subcloned into the retroviral vector $\mathrm{pFB}-\mathrm{Neo}$ (Stratagene) and transfected into ecotropic packaging cells (Phoenix) with the use of FuGENE transfection reagent (Roche Molecular Biochemicals). After 48 hours, retroviral supernatants were harvested and used to transduce NIH3T3 cells in the presence of Polybrene (Sigma), at a concentration of $5 \mu \mathrm{g}$ per milliliter. A binding assay for zymosan was also performed, as previously described. ${ }^{21}$

\section{RESULTS}

\section{PATIENTS AND PEDIGREE}

During the first phase of the screening, we identified a patient (the index patient) who had recurrent vulvovaginal candidiasis and had cells that were hyporesponsive to $C$. albicans stimulation, defined as cytokine production that was $15 \%$ or less of that stimulated by C. albicans in cells from healthy volunteers. The lack of cytokine production was pinpointed to an impaired response to $\beta$-glucan, indicating a potential defect in dectin-1 recognition.

To test this hypothesis, we sequenced the dectin- 1 gene in the patient. All six exons of dectin-1 gene, and the nearby intronic regions, were sequenced (see Table 1 in the Supplementary Appendix). We identified a homozygous SNP in exon 6 that caused a change of amino acid 238 from 


\begin{tabular}{|c|c|c|c|c|c|}
\hline Characteristic & $\begin{array}{l}\text { Index Patient } \\
\text { (Female) }\end{array}$ & Sister & Sister & Mother & Father \\
\hline Dectin-1 nucleotide genotype* & $x / x$ & $x / x$ & $x / x$ & $\mathrm{X} / \mathrm{Tyr}$ & X/Tyr \\
\hline Age (yr) & 25 & 27 & 23 & 53 & 58 \\
\hline Age at onset of symptoms (yr) & 10 & 10 & 12 & 40 & 55 \\
\hline Fingers and toes affected & $\begin{array}{l}\text { All fingers and } 4 \\
\text { toes, chronically }\end{array}$ & $\begin{array}{l}\text { All fingers and toes, } \\
\text { chronically }\end{array}$ & $\begin{array}{l}3 \text { Fingers and } 5 \\
\text { toes, transiently }\end{array}$ & $\begin{array}{l}\text { All fingers and toes, } \\
\text { chronically }\end{array}$ & $\begin{array}{l}4 \text { Toes partially affect- } \\
\text { ed, with complete } \\
\text { recovery }\end{array}$ \\
\hline $\begin{array}{l}\text { Recurrent vulvovaginal } \\
\text { candidiasis }\end{array}$ & Yes & No & Yes & No & Not applicable \\
\hline
\end{tabular}

$* X$ denotes the presence of a stop codon, rather than tyrosine (Tyr), at amino acid 238.

tyrosine to a stop codon (Tyr238X), leading to the loss of the last nine amino acids of the carbohydrate-recognition domain (Fig. 1A). Additional clinical questioning revealed that one of the patient's two sisters also had recurrent vulvovaginal candidiasis and both sisters had onychomycosis. The mother of the patient also had chronic onychomycosis, whereas the father had had only transient onychomycosis, with a relatively late age at onset and a complete recovery. The nucleotide sequence of wild-type dectin- 1 in one healthy volunteer and the nucleotide change $(\mathrm{A} \rightarrow \mathrm{C})$ in exon 6 in two persons who were heterozygous or homozygous for the stop mutation are shown in Figure S3A in the Supplementary Appendix.

The clinical characteristics of the patients are presented in Table 1. Microbiologic assessment of the nails of the three patients who were homozygous for the dectin- 1 mutation revealed growth of Trichophyton rubrum. The patients had no known predisposing factors, such as diabetes mellitus or infection with the human immunodeficiency virus. Genetic analysis revealed that both sisters of the patient were homozygous for the Tyr238X mutation, whereas the parents were heterozygous (Fig. 1B). The family members were white persons of Dutch ancestry, according to self-report, and the parents were not known to be related. Investigation of the human dectin-1 protein structure containing the early stop codon (Fig. S3B in the Supplementary Appendix) revealed that a cysteine disulfide bridge between the helix and the deleted strand was absent, a finding likely to have important functional consequences.

\section{IMMUNOLOGIC DEFECTS}

In the patients who had the early-stop-codon mutation, both monocytes (Fig. 2A) and macrophag- es (not shown) had significant defects in the production of interleukin- 6 after stimulation with $\beta$-glucan for 4 hours ( $\mathrm{P}=0.04$ for monocytes). Similar defects were apparent after stimulation of cells with either heat-killed or live C. albicans yeast (Fig. S1A in the Supplementary Appendix). The cytokine response was also defective after 24 hours of stimulation with heat-killed C. albicans hyphae (with a 25\% reduction of tumor-necrosisfactor production and a 34\% reduction of interleukin-6 release). In addition, cells from dectin1-deficient patients had a marked reduction in interleukin-17 production as compared with cells from persons with the dectin-1 wild-type allele (Fig. S4A in the Supplementary Appendix). Heterozygotes had intermediate production of proinflammatory cytokines on stimulation with $C$. albicans or $\beta$-glucan. In contrast, the response of the patients' cells to TLR stimuli, such as lipopolysaccharide or lipopeptides, was normal (Fig. S1B in the Supplementary Appendix). In addition, the amplification effect of the interaction between $\beta$-glucan and dectin- 1 on TLR2 stimulation of cytokines $^{7}$ was absent in persons who were homozygous for the mutation (Fig. S1C in the Supplementary Appendix).

To demonstrate that the defect in $\beta$-glucan recognition in the patients was indeed due to the truncated dectin-1 variant, we constructed vectors for both wild-type and mutated isoforms A and B of dectin- 1 and transfected them into NIH3T3 cells. These experiments revealed that neither mutated isoform A nor mutated isoform B could mediate $\beta$-glucan binding, in contrast to the wildtype receptors (Fig. S4B in the Supplementary Appendix). Moreover, although the wild-type dectin-1 isoforms A and B were normally expressed, the mutated isoforms were associated with sig- 


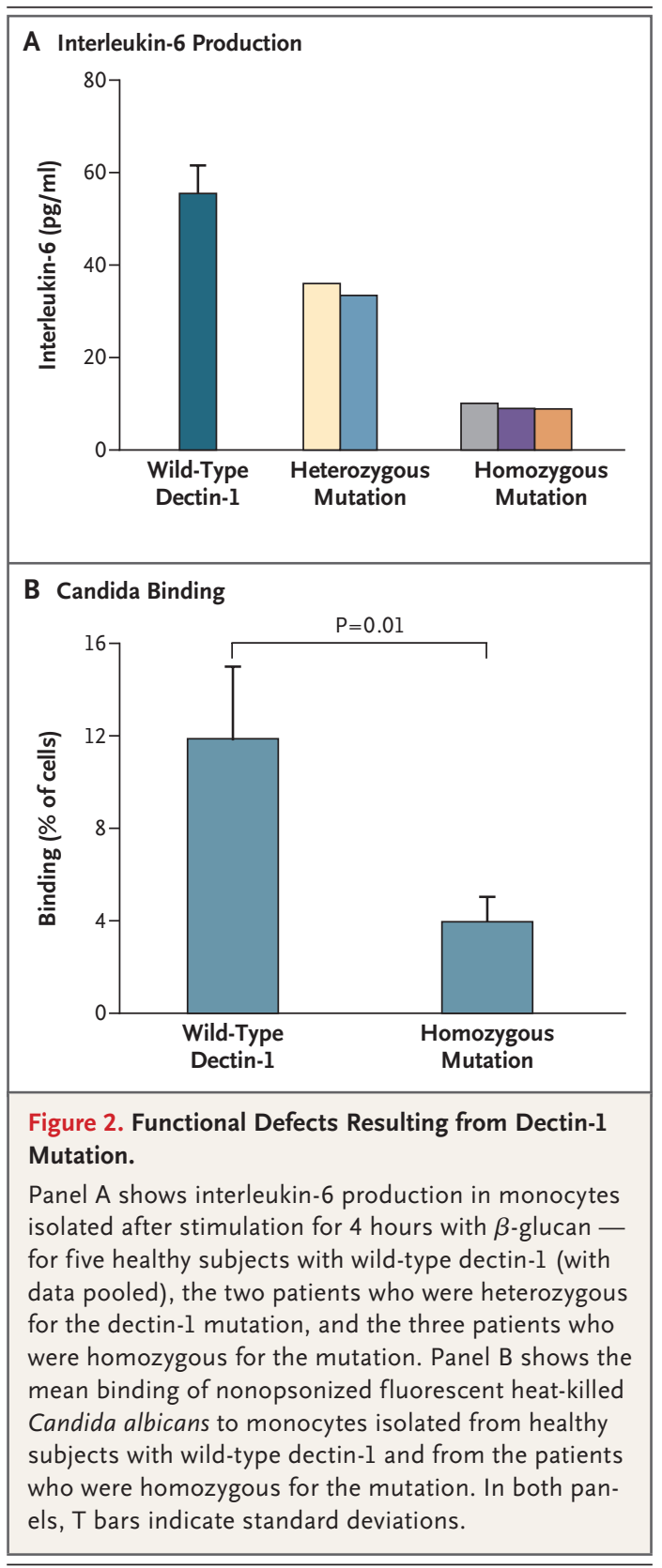

nificantly lower expression on the cell surface of the transfected cells (Fig. S4C in the Supplementary Appendix).

The in vitro data were corroborated in freshly isolated cells from the patients bearing the stopcodon mutation. Messenger RNA production by cells with the dectin- 1 isoforms was similar in persons who were homozygous for the wild-type allele and the patients who were homozygous for the stop mutation (Fig. S4D in the Supplementary Appendix). In contrast, monocytes and neutrophils from the patients who were homozygous for the stop-codon polymorphism lacked cell-membrane expression of dectin-1 (Fig. S4E in the Supplementary Appendix). Heterozygotes had intermediate expression of dectin-1.

The binding of heat-killed fluorescence-labeled C. albicans, which has high levels of $\beta$-glucan, ${ }^{22}$ to monocytes was significantly lower in the patients who were homozygous for the mutation than in healthy controls (Fig. 2B). However, phagocytosis of live C. albicans was normal in both monocytes and neutrophils from the patients who were homozygous for the stop codon (Fig. S2 in the Supplementary Appendix), showing the importance of alternative receptors for phagocytosis of live yeasts (other lectinlike receptors and TLRs). ${ }^{4}$ Monocytes and neutrophils from the patients who were homozygous for the mutation were as effective at killing C. albicans as were cells from normal persons (Fig. S2 in the Supplementary Appendix).

\section{FREQUENCY OF THE MUTATION IN POPULATIONS}

To determine whether the dectin-1 stop mutation was occurring in a phylogenetically conserved site, we compared the amino acid sequence of dectin- 1 among several mammals (Fig. S5A in the Supplementary Appendix). This investigation revealed that the mutation lies in an evolutionarily conserved region within the mammalian lineage. We then investigated the prevalence of the mutation in various human populations, and we genotyped persons from four cohorts of healthy persons of various ethnic groups, each representing populations from the major continents: 138 whites of Dutch ancestry (Europe), 99 people from Tanzania (Africa), 100 Han Chinese (Asia), and 105 Trio Indians (Native Americans) from Surinam (South America). Dectin-1 was sequenced completely in the Dutch population, with Tyr238X being the only nonsynonymous mutation identified. The Dutch population had an allele frequency of 0.069 , and the Tanzanian population, 0.035; all persons with the mutation were heterozygotes. The mutation was absent in the populations from China and Surinam. This variant has been recorded in the dbSNP database, as rs16910526.

A Human Genome Diversity Project selection browser was used to obtain further information about the frequency and world distribution of the SNP; this analysis confirmed our findings (Fig. 3). We analyzed haplotype diversity within a white population and a black population by studying 


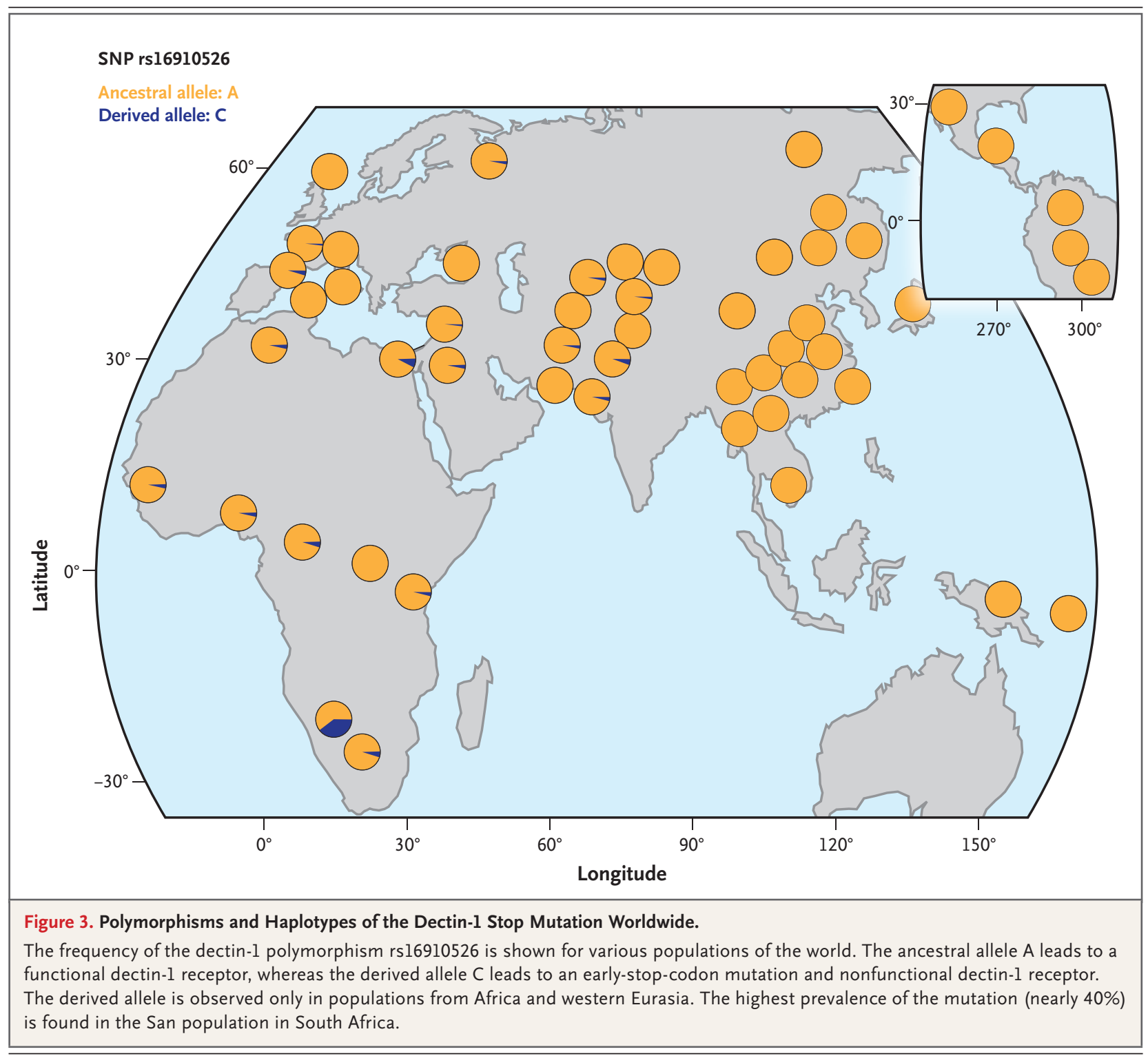

genomewide SNP data sets for samples obtained from 1422 whites from the U.K. 1958 birth cohort $^{13}$ and 171 African Maasai from Kinyawa, Kenya (from HapMap Phase III). ${ }^{14}$ Allele frequencies of the polymorphism within both populations did not differ significantly from those reported previously (whites, 0.078 , and blacks, 0.023 , as measured in our populations). ${ }^{13}$ Minimal mutationnetwork analysis revealed that, among the 23 haplotypes found in both populations, only 2 contain the stop mutation (Fig. SB in the Supplementary Appendix). Both haplotypes are present in both the European and African populations, and therefore are not population specific. In addition, the haplotypes are located near the ancient haplotype in the network, indicating that they are closely related to the ancient haplotype (Fig. S5C in the Supplementary Appendix).

\section{DISCUSSION}

Defective surface expression of dectin-1 due to the Tyr238X polymorphism results in lack of $\beta$-glucan recognition and an impaired cytokine response by monocytes and macrophages but normal killing of $C$. albicans by neutrophils. These data show the important role of $\beta$-glucan-dectin- 1 pathways for normal activation of the cytokine response, ${ }^{23}$ 
but also show the redundant nature of dectin- 1 in the phagocytosis and killing of Candida albicans. The normal function of neutrophils in persons in whom dectin-1 function is absent provides protection against invasive fungal infection. In contrast, the defective function of monocytes and macrophages with regard to cytokine release in the patients who were homozygous for the Tyr238X dectin-1 mutation is the most likely cause of the clinical phenotype characterized by mucocutaneous fungal infections.

Dysregulation of cytokine profiles is typically seen in patients with mucocutaneous fungal infections such as recurrent vulvovaginal candidiasis. ${ }^{24}$ Dectin- 1 is expressed on epithelial cells, and proinflammatory cytokines have been reported to be secreted by vaginal epithelial cells ${ }^{25}$ and to increase the natural antifungal activity of these cells. ${ }^{26}$ It is therefore conceivable that dectin- 1 function on epithelial cells would also be defective in our patients. Our data showing that a complete deficiency of dectin- 1 is accompanied by mucosal fungal infections are in line with a recent study showing the susceptibility of dectin-1-knockout mice to mucosal candidiasis. ${ }^{27}$

Dectin- 1 is also important for the development of the responses of type 17 helper T cells, ${ }^{28}$ and interleukin-17 production in our patients with defective dectin-1, as compared with healthy subjects, was reduced by $50 \%$ to $80 \%$ (Fig. S4A in the Supplementary Appendix). A clinical syndrome that closely resembles the syndrome in our family, with regard to fungal complications, is the hyper-IgE syndrome. Patients with the hyper-IgE syndrome have a defect in interleukin-17 production in response to infections with Staphylococcus aureus (cutaneous and pulmonary infection) and C. albicans (onychomycosis and mucosal infection). ${ }^{29,30}$ Our family had an isolated interleukin-17 defect in response to C. albicans stimulation, and it is tempting to speculate that this is the cause of the clinical picture of fungal infections in the patients. Our findings are strengthened by the description in this issue of the Journal of a family with a mutation of CARD9, the adaptor molecule for dectin-1, who presented with a phenotype practically identical to that of our family. ${ }^{31}$

Finally, the finding of this dectin-1 mutation in persons from both Europe and Africa leads to additional hypotheses. First, the identification of this polymorphism in all African populations assessed (including the San population) suggests that this is an ancient mutation that most likely emerged more than 60,000 years ago, before the split of the modern human populations in the late Paleolithic. ${ }^{32}$ This hypothesis is supported by the finding that the location of the haplotypes containing the Tyr238X mutation was close to the ancient haplotype. Second, the relatively high prevalence of the polymorphism in these populations may represent an important genetic susceptibility factor for mucosal fungal infection.

Supported by grants from the Netherlands Organization for Scientific Research (a Vidi Grant to Dr. Netea and Veni Grants to Drs. Cambi and van Spriel), the Wellcome Trust (to Dr. Brown), and the Dutch Cancer Society (2007-3917, to Dr. van Spriel). The use of genotypes from the British 1958 birth cohort collection was funded by grants from the U.K. Medical Research Council (G0000934) and the Wellcome Trust (068545/Z/02).

Dr. Johnson reports receiving grant support from Merck, consulting fees from Abbott Laboratories, and lecture fees from Kaplan Medical, Schering-Plough, Basilea Pharmaceutica, and Enzon; and Dr. Perfect, grant support from Pfizer and ScheringPlough, consulting fees from Enzon, Merck, Schering-Plough, Astellas, and Pfizer, and lecture fees from Enzon, Merck, Schering-Plough, Astellas, and Pfizer. No other potential conflict of interest relevant to this article was reported.
REFERENCES

1. Sobel JD. Vulvovaginal candidosis. Lancet 2007;369:1961-71.

2. Scher RK, Tavakkol A, Sigurgeirsson $\mathrm{B}$, et al. Onychomycosis: diagnosis and definition of cure. J Am Acad Dermatol 2007;56:939-44.

3. Spellberg BJ, Filler SG, Edwards JE Jr. Current treatment strategies for disseminated candidiasis. Clin Infect Dis 2006; 42:244-51.

4. Netea MG, Brown GD, Kullberg BJ, Gow NA. An integrated model of the recognition of Candida albicans by the innate immune system. Nat Rev Microbiol 2008;6:67-78.

5. Netea MG, Gow NA, Munro CA, et al. Immune sensing of Candida albicans re- quires cooperative recognition of mannans and glucans by lectin and Toll-like receptors. J Clin Invest 2006;116:1642-50.

6. Jouault T, Ibata-Ombetta S, Takeuchi $\mathrm{O}$, et al. Candida albicans phospholipomannan is sensed through Toll-like receptors. J Infect Dis 2003;188:165-72.

7. Brown GD, Herre J, Williams DL, Willment JA, Marshall AS, Gordon S. Dectin-1 mediates the biological effects of betaglucans. J Exp Med 2003;197:1119-24.

8. Gantner BN, Simmons RM, Canavera SJ, Akira S, Underhill DM. Collaborative induction of inflammatory responses by dectin-1 and Toll-like receptor 2. J Exp Med 2003;197:1107-17.

9. Taylor PR, Tsoni SV, Willment JA, et al. Dectin-1 is required for beta-glucan recognition and control of fungal infection. Nat Immunol 2007;8:31-8.

10. Saijo S, Fujikado N, Furuta T, et al. Dectin-1 is required for host defense against Pneumocystis carinii but not against Candida albicans. Nat Immunol 2007;8:39-46.

11. Dennehy KM, Ferwerda G, Faro-Trindade I, et al. Syk kinase is required for collaborative cytokine production induced through Dectin-1 and Toll-like receptors. Eur J Immunol 2008;38:500-6.

12. Gow NA, Netea MG, Munro CA, et al. Immune recognition of Candida albicans beta-glucan by dectin-1. J Infect Dis 2007; 196:1565-71

13. van Heel DA, Franke L, Hunt KA, et al. 
A genome-wide association study for celiac disease identifies risk variants in the region harboring IL2 and IL21. Nat Genet 2007;39:827-9.

14. Frazer KA, Ballinger DG, Cox DR, et al. A second generation human haplotype map of over 3.1 million SNPs. Nature 2007;449:851-61.

15. Browning SR, Browning BL. Rapid and accurate haplotype phasing and missing-data inference for whole-genome as sociation studies by use of localized haplotype clustering. Am J Hum Genet 2007; 81:1084-97.

16. Bandelt HJ, Forster P, Rohl A. Medianjoining networks for inferring intraspecific phylogenies. Mol Biol Evol 1999;16: 37-48.

17. Krieger E, Koraimann G, Vriend G. Increasing the precision of comparative models with YASARA NOVA - a selfparameterizing force field. Proteins 2002; 47:393-402.

18. Willment JA, Marshall AS, Reid DM, et al. The human beta-glucan receptor is widely expressed and functionally equivalent to murine Dectin-1 on primary cells. Eur J Immunol 2005;35:1539-47.

19. Cambi A, Gijzen K, de Vries JM, et al The C-type lectin DC-SIGN (CD209) is an antigen-uptake receptor for Candida albicans on dendritic cells. Eur J Immunol 2003;33:532-8
20. Vonk AG, Wieland CW, Netea MG, Kullberg BJ. Phagocytosis and intracellular killing of Candida albicans blastoconidia by neutrophils and macrophages: a comparison of different microbiological test systems. J Microbiol Methods 2002; 49:55-62.

21. Willment JA, Gordon S, Brown GD. Characterization of the human beta-glucan receptor and its alternatively spliced isoforms. J Biol Chem 2001;276:4381823.

22. Gantner BN, Simmons RM, Underhill DM. Dectin-1 mediates macrophage recognition of Candida albicans yeasts but not filaments. EMBO J 2005;24:1277-86. 23. Brown GD. Dectin-1: a signalling nonTLR pattern-recognition receptor. Nat Rev Immunol 2006;6:33-43.

24. Babula O, Lazdāne G, Kroica J, Linhares IM, Ledger WJ, Witkin SS. Frequency of interleukin-4 (IL-4) -589 gene polymorphism and vaginal concentrations of

IL-4, nitric oxide, and mannose-binding lectin in women with recurrent vulvovaginal candidiasis. Clin Infect Dis 2005;40: 1258-62.

25. Steele C, Fidel PL Jr. Cytokine and chemokine production by human oral and vaginal epithelial cells in response to Candida albicans. Infect Immun 2002;70: 577-83.

26. Barousse MM, Steele C, Dunlap K, et Copyright (c) 2009 Massachusetts Medical Society. al. Growth inhibition of Candida albicans by human vaginal epithelial cells. J Infect Dis 2001;184:1489-93.

27. Hise AG, Tomalka J, Ganesan S, et al. An essential role for the NLRP3 inflammasome in host defense against the human fungal pathogen Candida albicans. Cell Host Microbe 2009;5:487-97.

28. LeibundGut-Landmann S, Gross O, Robinson MJ, et al. Syk- and CARD9-dependent coupling of innate immunity to the induction of $\mathrm{T}$ helper cells that produce interleukin 17. Nat Immunol 2007;8: 630-8.

29. Grimbacher B, Holland SM, Gallin JI, et al. Hyper-IgE syndrome with recurrent infections - an autosomal dominant multisystem disorder. N Engl J Med 1999; 340:692-702.

30. Milner JD, Brenchley JM, Laurence A et al. Impaired $\mathrm{T}(\mathrm{H}) 17$ cell differentiation in subjects with autosomal dominant hyper-IgE syndrome. Nature 2008;452:7736.

31. Glocker E-O, Hennigs A, Nabavi M, et al. A homozygous CARD9 mutation in a family with susceptibility to fungal infections. N Engl J Med 2009;361:1727-35.

32. Underhill PA, Shen P, Lin AA, et al. Y chromosome sequence variation and the history of human populations. Nat Genet 2000;26:358-61.

POWERPOINT SLIDES OF JOURNAL FIGURES AND TABLES

At the Journal's Web site, subscribers can automatically create PowerPoint slides. In a figure or table in the full-text version of any article at NEJM.org, click on Get PowerPoint Slide. A PowerPoint slide containing the image, with its title and reference citation, can then be downloaded and saved. 